\title{
An uncommon cause of facial pain
}

Kosar Hussain, Muhammad Hassan, Sara Hussain

\section{CASE}

A woman aged 42 years presented with left-sided facial pain. Her symptoms had begun three weeks prior. She initially experienced a sensation of 'fullness' around the left eye and maxillary region. A week ago, she received symptomatic treatment for sinusitis, along with a course of oral antibiotic. However, her symptoms progressed and increased in severity. She had now developed frequent episodes of sharp left-sided facial pain. She also reported blurring of vision in her left eye with associated diplopia.

Her past medical history was significant for left thigh myxoid liposarcoma that was diagnosed four years ago. She had received primary neoadjuvant radiotherapy, followed by surgical resection of the tumour. She has been in remission since. Her last oncology follow-up was one year ago.

On examination, her vital signs were all within normal range. Her left eye was found to be congested with surrounding lid oedema. There was a partial ptosis in addition to weakness of the oculomotor, trochlear and abducens nerves. There was pain with left eye movement. The pupil was in mid-position and nonreactive. Visual acuity was normal in both eyes, with full visual fields to confrontation. Fundoscopy was normal. There was associated reduced sensation in the $\mathrm{V} 1$ and $\mathrm{V} 2$ divisions of the trigeminal nerve. The remaining systemic examination was unremarkable.
QUESTION 1

What is the differential diagnosis of facial pain?

QUESTION 2

What is painful ophthalmoplegia?

QUESTION 3

What are the cavernous sinuses?

\section{QUESTION 4}

What are the features of cavernous sinus syndrome (CSS)? What are the causes of CSS?

\section{ANSWER 1}

The differential diagnosis of facial pain can be broadly categorised as follows. ${ }^{1}$

- Neuralgias - paroxysmal pain that has a sharp, shock-like quality that occurs in the distribution of a nerve. A classic example is trigeminal neuralgia.

- Facial pain syndromes with cranial nerve symptoms and signs - facial pain accompanied by lesions of one or more cranial nerves. Examples of conditions that affect the vision include optic neuritis, giant cell arteritis and syndromes of painful ophthalmoplegias.

- Trigeminal autonomic cephalalgias primary headache syndromes associated with signs of cranial autonomic activation. A classic example is cluster headaches.

- Pure facial pain without neurological signs - includes pain arising from the sinuses, ear, temporomandibular joint and oral cavity.

- Primary headache disorders - primary headache disorders such as migraine may also present as facial pain. They can generally be diagnosed clinically with careful attention to history and examination findings.

\section{ANSWER 2}

Painful ophthalmoplegia is characterised by pain localised in the eye, orbit or forehead with ipsilateral ocular palsies. It arises from various pathologies localised in the orbit, the tip of the orbit, the cavernous sinus or the subarachnoid space. ${ }^{2}$

\section{ANSWER 3}

Cavernous sinuses are dural venous sinuses located on either side of the pituitary fossa. They contain the internal carotid artery, oculomotor nerve, trochlear nerve, abducens nerve, and the ophthalmic and maxillary division of the trigeminal nerve.

\section{ANSWER 4}

Painful ophthalmoplegia is the hallmark of CSS. It manifests by weakness of the cranial nerves III, IV and VI, and numbness in the $\mathrm{V} 1$ and $\mathrm{V} 2$ divisions of the trigeminal nerve. Causes of CSS include the following.

- Neoplasms - primary tumours that can invade the cavernous sinus include pituitary adenoma and meningioma. ${ }^{3}$ Metastasis to the cavernous sinus is uncommon. ${ }^{3}$ Most common primary sites for metastasis are the head and neck. However, spread from distant lesions such as kidney, liver, lung, colon and prostate cancers have also been reported. ${ }^{4}$

- Vascular - aneurysms, arteriovenous malformation and carotid-cavernous thrombosis. 


\section{- Inflammatory-}

- non-specific inflammatory causes (eg Tolosa-Hunt syndrome)

- specific inflammatory causes (eg sarcoidosis, systemic lupus erythematosus, granulomatosis with polyangiitis).

- Infectious - invasive bacterial, fungal or viral infections.

\section{CASE CONTINUED}

The patient was sent for an urgent computed tomography scan of the brain, which revealed a hypodense mass lesion in the middle cranial fossa with post-contrast enhancement. The left cavernous sinus did not enhance with contrast. There was no radiological evidence of significant mass effect.

The patient was sent urgently to the local hospital for further management. Magnetic resonance imaging of the brain was performed, which showed a large, aggressive mass involving the left middle cranial fossa, cavernous sinus, left orbital apex and maxillary sinuses (Figure 1).

During the patient's stay in hospital, further imaging revealed widespread metastasis to the lung pleura, right psoas and lumbosacral region. She was offered palliative radiotherapy to the brain and lumbosacral region and a course of steroids.

\section{Key points}

- Facial pain with cranial nerve involvement is almost exclusively of secondary origin. Imaging is warranted in most cases.

- Cavernous sinus syndrome is characterised by painful ophthalmoplegia. It is secondary to an underlying structural lesion and requires prompt recognition.

- Be mindful of the possibility of new metastatic lesions in a patient with a past or current history of malignancy.

- Common diagnoses are often the most likely diagnosis. Sometimes, however, the rare diagnosis is the correct one.

\section{Authors}

Kosar Hussain MBBCh, Medical Registrar General Medicine, Goulburn Valley Health, Vic. dr.kosar.hussain@gmail.com

Muhammad Hassan MBBS, General Practitioner, Tatura, Vic

Sara Hussain MBBCh, Emergency Trainee, Rashid Hospital, Dubai Health Authority, Dubai, UAE

Competing interests: None.

Funding: None.

Provenance and peer review: Not commissioned, externally peer reviewed.

\section{References}

1. Siccoli MM, Bassetti CL, Sándor PS. Facial pain: Clinical differential diagnosis. Lancet Neurol 2006;5(3):257-67. doi: 10.1016/S14744422(06)70375-1.

2. Kline LB, Hoyt WF. The Tolosa-Hunt syndrome. J Neurol Neurosurg Psychiatry 2001;71(5):577-82.

3. Fernández S, Godino O, Martínez-Yélamos S, et al. Cavernous sinus syndrome: A series of 126 patients. Medicine (Baltimore) 2007;86(5):278-81. doi: 10.1097/MD.0b013e318156c67f.

4. Kahlon A, Singh K, Jain S, Singh G. Acute cavernous sinus syndrome due to metastatic infiltration of the cavernous sinus and clivus: Unknown primary. European Geriatric Medicine 2016;5(7):471-73. doi: 10.1016/j eurger.2016.06.005

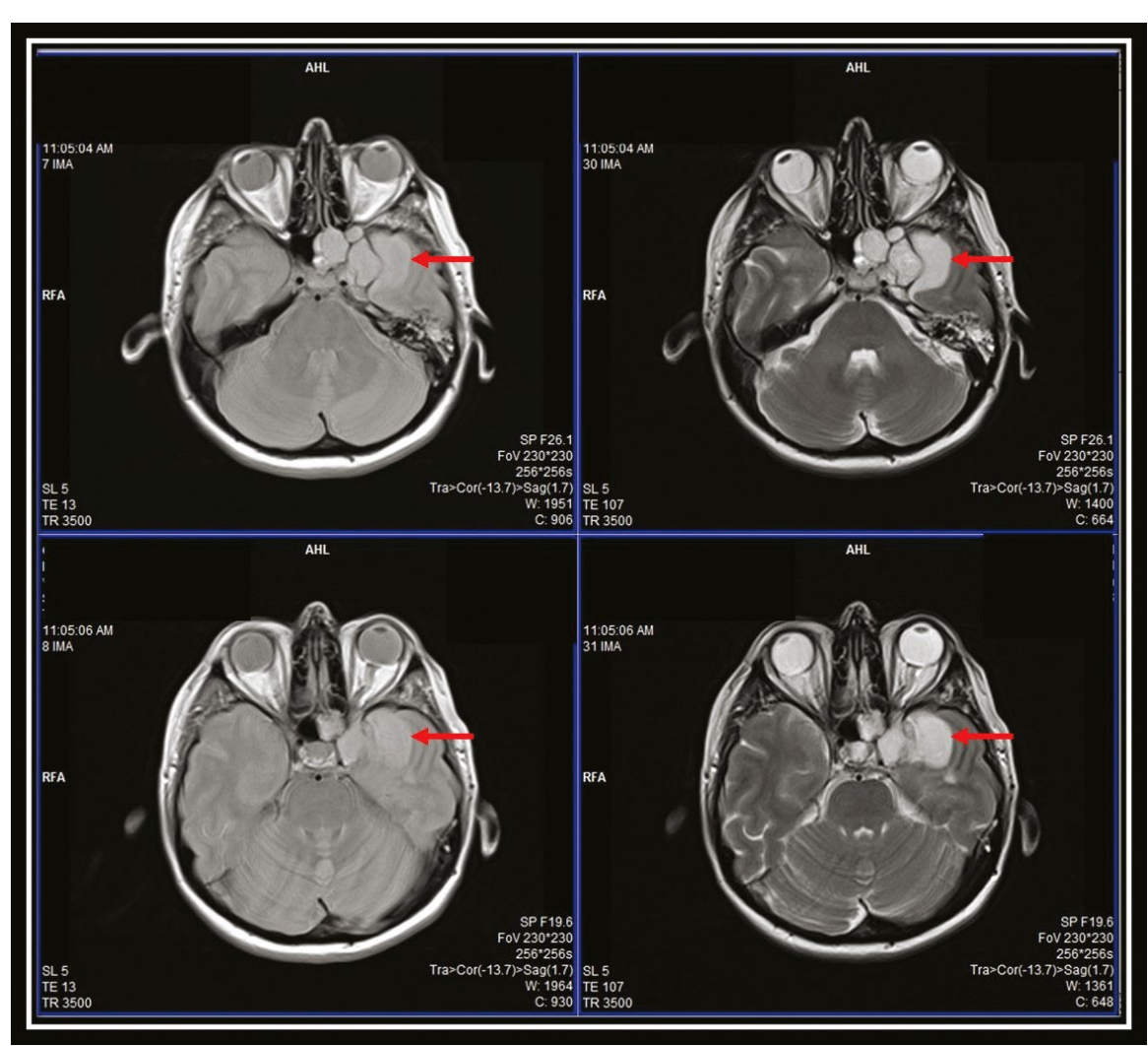

Figure 1. Magnetic resonance imaging of the brain showing a large, aggressive mass involving the left middle cranial fossa, cavernous sinus, left orbital apex and maxillary sinuses 